\title{
Ohmic electromechanical dissipation in nanomechanical cantilevers
}

\author{
T. Barois, A. Ayari, A. Siria, S. Perisanu, P. Vincent, P. Poncharal, and S. T. Purcell \\ Laboratoire de Physique de la Matière Condensée et Nanostructures (UMR CNRS 5586), Université Claude Bernard, \\ F-69622 Villeurbanne, France
}

(Received 16 December 2011; published 10 February 2012)

\begin{abstract}
We study the contribution of ohmic dissipation to the mechanical damping of nanoresonators. This damping occurs when DC voltage is applied to a resistive resonator, because the mechanical motion modifies the associated capacitance, thus inducing a dissipative current. Silicon carbide nanowire resonators were studied as a function of applied voltage and their geometrical environment. Nanometric positioners were used to control and continuously modify the position of the resonator with respect to counter electrodes. The experimental results are shown to be in agreement with an electromechanical model developed here, which allows for the establishment of a universal formula for the lower dissipation limit of a nanoresonator in its capacitive environment.
\end{abstract}

DOI: 10.1103/PhysRevB.85.075407

PACS number(s): 81.07.Oj, 78.67.Uh, 62.25.-g

\section{INTRODUCTION}

Resonating nanoelectromechanical systems (NEMS) have been intensively studied as sensors due to their very high sensitivity to local environments. ${ }^{1-3}$ NEMS based measurements often consist of tracking the frequency shifts of the resonator when it is perturbed by external forces. The accuracy with which the frequency is measured is inversely proportional to dissipation $^{4,5}$ which also controls power consumption. The search for high quality factor resonators has lead to much work on the dissipation mechanisms in such resonators. Though fundamental limits have been proposed for the intrinsic dissipation of nanocantilevers in terms of thermoelastic damping and phonon-phonon coupling, ${ }^{6-8}$ additional experimental dissipation processes can increase the intrinsic damping, such as air friction, ${ }^{9}$ clamping losses, ${ }^{10}$ external electrical circuits coupling, ${ }^{11,12}$ and ohmic dissipation. The latter is the subject of this article. Ohmic losses, or "electrostatic damping," are the results of the current induced in a series resistance by the electrostatic loading of the capacitance made by the oscillating resonator and its environment.

The role of the electrical current in the dissipation of electromechanical resonators has been discussed for piezoelectrical systems where charge flow is induced by the transducer deformation (see Ref. 13 for a list of references). This process is termed "passive shunt damping,"14 where an external impedance dissipates the piezoelectrical induced current. In nanomechanical devices, a comparable model can be found in Ref. 15, where the origin of the current flow is not piezoelectricity but the capacitance of the resonator with the environment under bias voltage. DC voltage is often used in NEMS devices to tune the mechanical resonances through the capacitance to higher ${ }^{16}$ or lower ${ }^{17}$ frequencies, but it can also increase the damping. ${ }^{18}$ Similar behaviors are found in transistor configurations, where the gate voltages needed for the transconductance induce static charges that can lead to significant dissipation.

We report experimental results on a silicon carbide singleclamped resonator where the electrostatic damping dominates. The results are analyzed with the aid of an analytical treatment of resonators capacitively coupled to their environment. Thanks to a bottom-up approach and in situ nanomanipulation, the damping was explored with respect to the capacitive environment as well as the bias voltage. A wide survey of results in the literature also shows how the dissipation varies with the relevant dimensionless parameters, the time ratio $T$ and coupling strength $\beta$ (see below). Our model sets a universal framework for electrostatic damping in micro- and nanoelectromechanical systems.

\section{EXPERIMENTAL SETUP}

A silicon carbide $(\mathrm{SiC})$ nanowire resonator was studied in a home-built high vacuum system $\left(\sim 10^{-9}\right.$ Torr $)$ including an in situ scanning electron microscope (Orsay Physics, e-CLIPSE) for imaging and measuring the mechanical resonance frequencies and amplitudes. The nanowire was glued onto a tungsten support tip which was manipulated near two types of counter electrodes. A rather full set of experiments were carried out with a gold counter electrode and a reduced set with a shorter $\mathrm{SiC}$ nanowire glued on a second tungsten tip (Fig. 1). Both the nanowire tungsten support and the counter electrodes were fixed on Attocube positioners (ANPxyz50) with $X Y Z$ relative displacement up to a nanometer precision. The $\mathrm{SiC}$ nanowire resonator was $200 \mu \mathrm{m}$ long and $250 \mathrm{~nm}$ thick. The tungsten tip was point soldered onto a wire heating loop used to anneal the nanowire resonator up to $800^{\circ} \mathrm{C}$. The annealing was used to degas the nanowire but it also progressively reduced its electrical resistance. ${ }^{19}$ The resonances were excited by piezoelectric actuation.

Two methods were used to estimate resistance values. First, the apex of the nanowire electrode was brought into contact with the gold counter electrode for two point measurements. This is subject to unstable and compromising contact resistances, which can even be nonlinear. The resistance $R$ of the nanowire was mostly estimated using a technique combining field emission and the tuning of the mechanical resonances by applied voltage. ${ }^{20}$ Field emission occurred when the electric field was high enough to extract electrons from the apex of the resonator. The resulting DC current $I_{\mathrm{FE}} \sim 1 \mathrm{nA}$ induced a voltage drop $\Delta V$ along the nanowire. The voltage drop reduced the tuning, which, knowing the emission current, can be used to estimate the resistance. This is detailed in Fig. 2 and its caption. Typical values for $R$ were in the range of $1-10 \mathrm{G} \Omega$. (A summary of resistance evolutions and measurements is given 


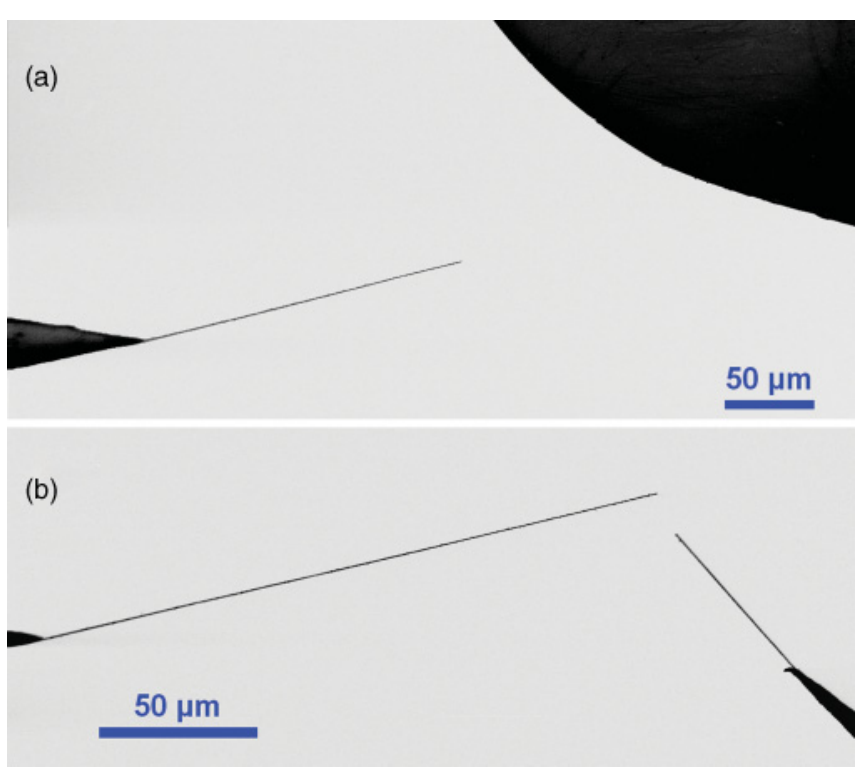

FIG. 1. (Color online) SEM images of the silicon carbide nanowire resonator (on the left in both images) on its tungsten tip in front of (a) the gold electrode and (b) the short nanowire.

in Table II.) Another important parameter is the capacitance and its dependence on the position of the nanowire. This will be discussed throughout the rest of the article.

\section{MODEL}

Consider the equation of motion for the first mode of the nanowire where $x$ is the amplitude at the apex. The equation of motion linearized around the equilibrium position is:

$$
m_{e} \frac{d^{2} x}{d t^{2}}+k\left(V_{\mathrm{DC}}\right) x=C^{\prime} V_{\mathrm{DC}} V_{\mathrm{NW}},
$$

where $m_{e}$ is the effective mass and $k\left(V_{\mathrm{DC}}\right)$ the restoring force for the first mode. $C(x)$ is the nanowire/electrode capacitance

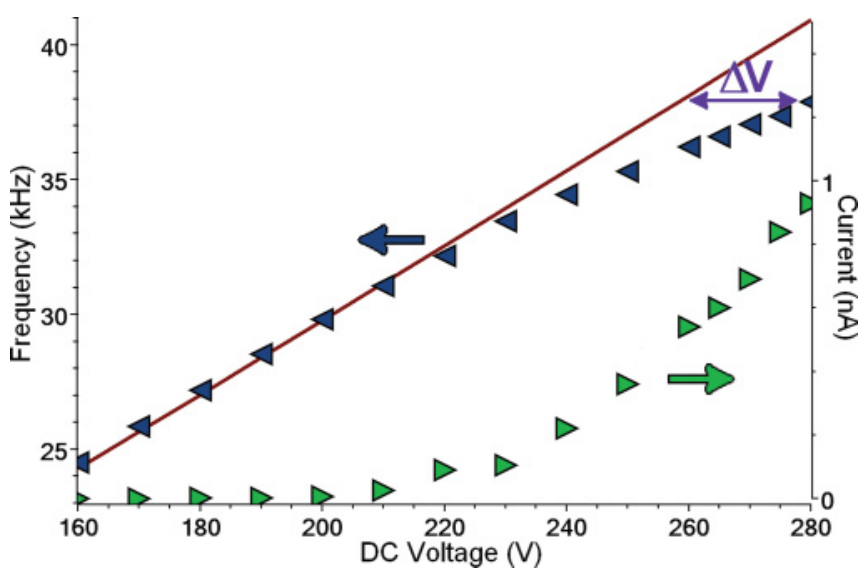

FIG. 2. (Color online) First mode resonant frequency (pointing left triangles, left axes) and field emission current (pointing right triangles, right axes) as a function of the applied DC voltage. The resonant frequencies vary linearly with the applied voltage for lower voltages, i.e., 100-200 V (continuous line). When field emission occurs, the resonant frequency shifts from the linear dependance. The voltage shift $\Delta V$ corresponds to the voltage drop along the nanowire. The resistance is $\Delta V / I_{\mathrm{FE}}$. for a given equilibrium position as a function $x, V_{\mathrm{DC}}$ is the applied DC voltage to the tungsten tip (with the counter electrode grounded), and $V_{\mathrm{NW}}$ is the time-dependant voltage along the nanowire (such that $V_{\mathrm{DC}}+V_{\mathrm{NW}}$ is the voltage at the apex). The force in Eq. (1) comes from the derivative of the electrostatic potential energy $C(x)\left(V_{\mathrm{DC}}+V_{\mathrm{NW}}\right)^{2} / 2$ with respect to $x$. $V_{\mathrm{NW}}$ is typically much smaller than $V_{\mathrm{DC}}$. A more detailed definition of the restoring force including DC voltage tuning could be given, but here a simple phenomenological form is used in order to concentrate on electrostatic dissipation. Pure mechanical dissipation is also neglected in this section. The equation for $V_{\mathrm{NW}}$, including the flow of electrical current through the resistive nanowire to the displacement-dependant capacitance $C(x)$, is

$$
-\frac{V_{\mathrm{NW}}}{R}=V_{\mathrm{DC}} C^{\prime} \frac{d x}{d t}+C \frac{d V_{\mathrm{NW}}}{d t},
$$

where $C^{\prime}$ is the derivative of $C$ with respect to $x$.

Equations (1) and (2) can be combined to give a third-order equation for the displacement:

$$
\frac{T}{\omega_{0}} \frac{d^{3} x}{d t^{3}}+\frac{d^{2} x}{d t^{2}}+\omega_{0} T(1+\beta) \frac{d x}{d t}+\omega_{0}^{2} x=0,
$$

where the two dimensionless parameters $T$ and $\beta$ have been introduced:

$$
\begin{aligned}
& T=R C \omega_{0} \\
& \beta=\frac{\left(C^{\prime} V_{\mathrm{DC}}\right)^{2} k^{-1}}{C} .
\end{aligned}
$$

The time parameter $T$ is the ratio of the mechanical and electrical time constants with $\omega_{0}$ the resonant angular frequency given by $\omega_{0}^{2}=k / m_{e} . \beta$, the "coupling strength," 15 is a second useful parameter, which is the ratio between the motional capacitance $C_{m}$ (discussed below) and the electrical capacitance $C$.

The characteristic polynomial $\mathcal{P}(z)$ associated with Eq. (3) gives complex solutions, i.e., damped oscillations where frequencies and damping rates correspond to the imaginary and real parts, respectively. As for a harmonic oscillator with damping, we introduce the electrostatic damping rate as $\Gamma_{\mathrm{es}}=2 \Re e\left(z_{ \pm}\right)$, where $z_{ \pm}$are the complex solutions of $\mathcal{P}(z)$. For $\beta \ll 1, z_{ \pm}$can be expressed to first order as

$$
z_{+}=i \omega_{0}+\omega_{0} \frac{i \beta T}{2 T-2 i} \quad \text { and } \quad z_{-}=\overline{z_{+}} .
$$

One gets a universal expression for the electrostatic damping rate:

$$
\Gamma_{\mathrm{es}}=\beta \omega_{0} \frac{T}{1+T^{2}} .
$$

A balance of energy argument was used to derive a similar expression in Ref. 15, which, however, underestimates $\Gamma_{\mathrm{es}}$ by a factor of $1 / \pi$. For completeness to first order in $\beta$, the angular resonance frequency also varies with $T$ and is given by $\Im m\left(z_{ \pm}\right)$. The damping rates and frequencies for the low $T$ (LT) and high $T$ (HT) regimes are:

for $T \ll 1$

$$
\begin{gathered}
\Gamma_{\mathrm{LT}}=\beta \omega_{0} T=\frac{R\left(C^{\prime} V_{\mathrm{DC}}\right)^{2}}{m_{e}} \\
\omega_{\mathrm{LT}}=\omega_{0}+R^{2} \frac{\omega_{0} C\left(C^{\prime} V_{\mathrm{DC}}\right)^{2}}{2 m_{e}},
\end{gathered}
$$


TABLE I. Motional dipoles for the equivalent electrical circuit. $\alpha \equiv C^{\prime} V_{\mathrm{DC}}$.

\begin{tabular}{lc}
\hline \hline Electromechanical representation & Electrical lumped model \\
\hline$q_{C}$ & $C^{\prime} V_{\mathrm{DC}} x$ \\
$L_{m}$ & $m / \alpha^{2}$ \\
$R_{m}$ & $\Gamma / \alpha^{2}$ \\
$C_{m}$ & $\alpha^{2} / k$ \\
\hline \hline
\end{tabular}

for $T \gg 1$

$$
\begin{gathered}
\Gamma_{\mathrm{HT}}=\frac{\beta \omega_{0}}{T}=\frac{G\left(C^{\prime} V_{\mathrm{DC}}\right)^{2}}{m_{e} \omega_{0}^{2} C^{2}} \\
\omega_{\mathrm{HT}}=\omega_{0} \sqrt{1+\beta}-G^{2} \frac{\left(C^{\prime} V_{\mathrm{DC}}\right)^{2}}{2 m_{e} \omega_{0}^{3} C^{3}},
\end{gathered}
$$

where $G=1 / R$ is the conductance. At low $T$ [Eqs. (6) ${ }^{18}$ and (7)], the charges move along the resonator as a dissipative current. At high $T$ [Eqs. (8) and (9)], the large $R C$ filtering blocks the dissipative current and consequently affects the restoring force only. This force is characterized by constant voltage in the lower limit and by constant charge in the higher limit. In the low $T$ regime, the damping rate is proportional to the resistance as expected for ohmic losses. In the high $T$ regime, the effect of $R C$ filtering is less intuitive since the dissipation increases with the conductance $G$ of the cantilever.

The electromechanical system has an equivalent $\mathrm{AC}$ circuit where the mechanical components are represented as motional dipoles $\left\{R_{m}, L_{m}, C_{m}\right\}$ (see Table I and Fig. 3). It is similar to the Butterworth-Van Dyke circuit for quartz resonators. ${ }^{21}$ For such resonators, the proportionality between charge and displacement is a physical property of piezoelectric materials, while here it is the result of static voltage loading of the position-dependant capacitance. In the low $T$ regime, the usual piezoelectric relation between charge and displacement is replaced by $q_{C}=x C^{\prime} V_{\mathrm{DC}}$.

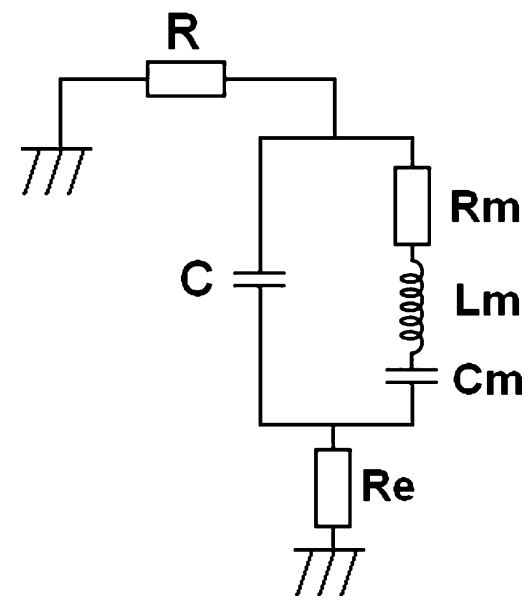

FIG. 3. Equivalent circuit for a single clamped resistive cantilever in a capacitive environment. The position $x$ of the cantilever is represented by the charge $q$ on the motional capacitance $C_{m}$ upper plate. $R_{m}$ corresponds to a purely mechanical damping neglected in the discussion of the model. $R$ is the resistance of the cantilever and $C$ the total capacitance with environment. $R_{e}$ is the resistance of the counter electrode (discussed below).

\section{EXPERIMENTAL RESULTS}

We concentrate on experimentally validating Eq. (6), which is for electrostatic damping in the low $T$ regime. The $7 \mathrm{kHz} \mathrm{SiC}$ nanowire was well adapted for this purpose. These resonators have in general two transverse polarizations for each mode. The first set of experiments was carried out with the gold electrode, as shown in Fig. 1(a). With a good axial alignment of the nanowire, only the polarization transverse to the image plane allowed a full range of measurements. The needed $R C$ time constant was determined from the exponential decay of the field emission current to a superimposed square voltage modulation $(\sim 1 \mathrm{~Hz})$. The frequency cutoff, $1 / 2 \pi R C \approx$ $50 \mathrm{kHz}$, was at least one order of magnitude higher than the resonance frequency.

Frequency spectra of the mechanical resonances were measured using the SEM electron beam in spot mode focused on the edge of the nanowire. The setup is similar to that in Ref. 22, and a lock-in SRS830 was used for the reconstruction of spectra from the secondary electron detector signal. In order to avoid self-sustained oscillation of the nanowire induced by the electron beam, ${ }^{23}$ the electron beam was focused close to the clamping of the nanowire. The damping rate and resonant frequency were both estimated from measurements of the resonator amplitude and the phase shift with respect to the piezoelectrical driving signal (see Fig. 4).

The first experiment was to study the influence of the nanowire/electrode geometry on damping through the spatial derivative of the capacitance. $C^{\prime}$ was varied by changing the relative angle between the nanowire and the electrode. The angle was varied from $\theta=0^{\circ}$ to $\theta=90^{\circ}$ (see Fig. 5) for a set voltage of $10 \mathrm{~V}$ and a constant apex/electrode distance $(d=39.7 \mu \mathrm{m} \pm 1 \mu \mathrm{m})$. At the resonant frequency, the amplitude is typically $1 \mu \mathrm{m}$, giving a capacitive current smaller than $1 \mathrm{pA}$. The important result is that the dissipation was a minimum when the oscillation of the nanowire was parallel to the electrode surface $\left(\theta=90^{\circ}\right)$ and increased as the oscillation became more perpendicular, reaching a maximum at $0^{\circ}$. Though a precise modeling of $C(x)$ is rather complicated for a wire/electrode geometry, it is clear that the parameter that modifies it mostly during an oscillation is the apex/electrode distance. This distance varies strongly for perpendicular oscillations and weakly for parallel oscillations.

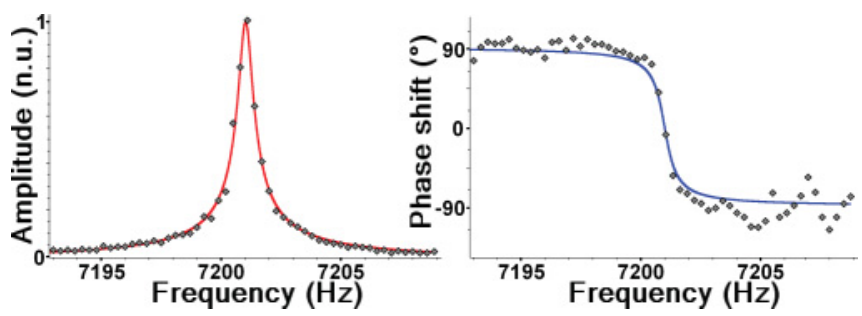

FIG. 4. (Color online) Mechanical resonance of the nanowire far from the counter electrode $(d>500 \mu \mathrm{m})$ without applied voltage. The electron beam was focused on the nanowire to detect amplitude and phase shift of the resonance. The intrinsic frequency for the first mode is $7201.04 \pm 0.02 \mathrm{~Hz}$ with a damping rate of $4.1 \pm 0.4 \mathrm{~s}^{-1}$, giving a $Q$ factor of $11000 \pm 1000$. Amplitude is in normalized units (n.u.). 


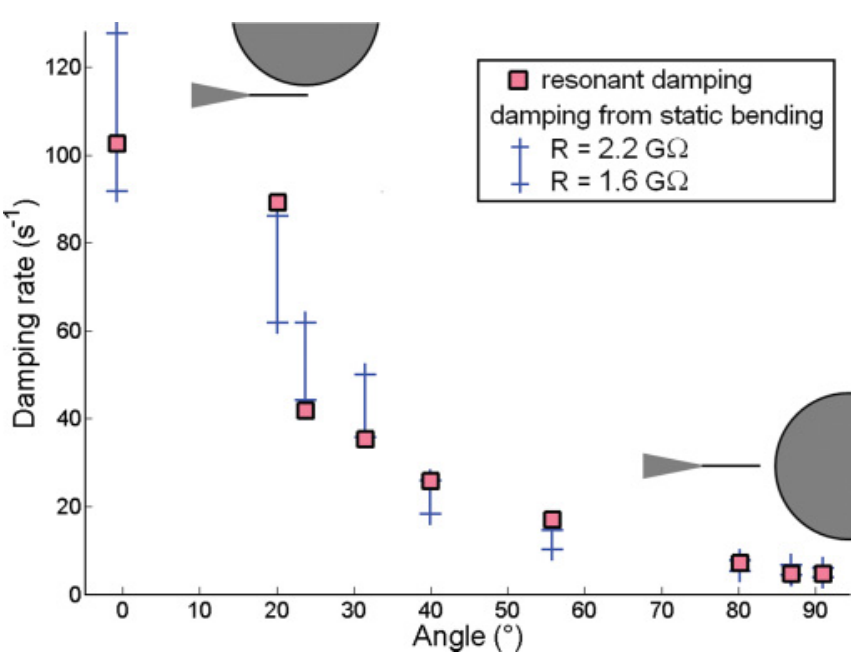

FIG. 5. (Color online) Damping rate from fitting the phase of the resonance spectra and by using the static bending method (see text). The DC voltage was $10 \mathrm{~V}$ and the nanowire/electrode distance was $d=39.7 \mu \mathrm{m}$. When $\theta=90^{\circ}$, the nanowire was perpendicular to the electrode, and when $\theta=0^{\circ}$ the nanowire and the electrode surface were parallel. The resonances were excited by piezomotive actuation. The error bars correspond to the electrostatic damping from Eq. (6) with $R=1.6 \mathrm{G} \Omega$ and $R=2.2 \mathrm{G} \Omega$, with a residual damping rate of $\Gamma_{\mathrm{ex}}=5 \mathrm{~s}^{-1}$. $C^{\prime}$ is estimated from an image analysis.

It is possible to give a quantitative estimate of $C^{\prime}$ from the image analysis of the bending $x_{b}$ of the nanowire with the applied DC voltage $V_{\mathrm{DC}}$. This was done almost simultaneously at each angle where a resonance was measured. Using the balance between mechanical restoring force and electrostatic force, $C^{\prime}$ is given by

$$
m_{e} \omega_{0}^{2} x_{b}=\frac{1}{2} C^{\prime} V_{\mathrm{DC}}^{2} .
$$

The restoring force acting on the static deflection is assumed to be the same as for the first mode. This is shown to be a good approximation, because the experimental nanowire shape under static deflection fits well to the first mode shape. The nanowire mass is estimated using the bulk $\mathrm{SiC}$ density and measured length and radius. From Eqs. (6) and (10), the damping rate can be expressed as

$$
\Gamma_{t}=\Gamma_{\mathrm{ex}}+4 m_{e} R\left(\frac{\omega_{0}^{2} x_{b}}{V_{\mathrm{DC}}}\right)^{2},
$$

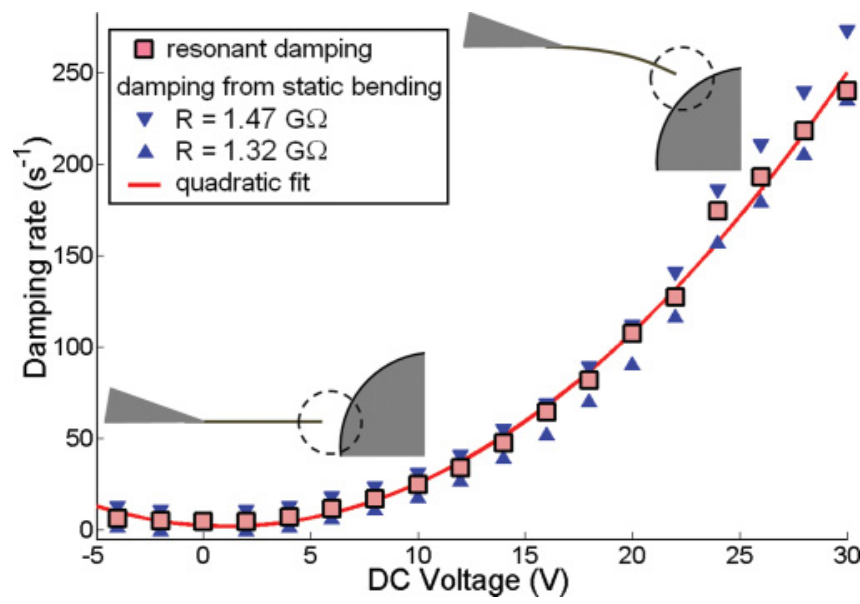

FIG. 6. (Color online) Damping rate from resonance spectra (squares). The electrostatic damping from Eq. (6) is plotted for $R=1.47 \mathrm{G} \Omega$ (down triangle) and $R=1.32 \mathrm{G} \Omega$ (up triangle). For each applied voltage, as the nanowire deflects, the tungsten nanowire support is moved to keep the local geometry of the apex/electrode constant (dashed circle line). An optimal fitting agreement is found for a nanowire resistance $R=1.39 \mathrm{G} \Omega$, a residual damping rate $\Gamma_{\mathrm{ex}}=3.7 \mathrm{~s}^{-1}$, and an effective mass $m_{e}=5 \cdot 10^{-15} \mathrm{~kg}$. Quadratic model shows a qualitative agreement with the data (solid line).

where $\Gamma_{\mathrm{ex}}$ is introduced to describe the extra sources of dissipation. Figure 5 shows that there is a good agreement between the measurements of the damping rate and the static bending converted using Eq. (11) for $R=1.9 \mathrm{G} \Omega$, in support of the electrostatic damping model. Note that for this work, $\Gamma_{\mathrm{es}}$ generally dominates $\Gamma_{\mathrm{ex}}$. There is a low value of $\Gamma_{t}$ at $\theta=90^{\circ}$. In principle, one expects a cancellation of the electrostatic damping for a perfectly symmetrical capacitive environment. Complementary work in a near-field geometry suggests that the effective cancellation of the capacitance derivative is not simple.

The second experiment was to measure the effect of a varying DC voltage on the dissipation with $C^{\prime}$ kept constant. The difficulty was that the nanowire bent with the applied DC voltage, which in turn varied $C^{\prime}$ through the change in the distance and the angle of the free end with respect to the counter electrode. To compensate for this effect, the tip was moved laterally at each DC voltage to keep both the distance and the relative angle constant. With this precaution, the dependance of the dissipation on voltage is quadratic as shown in Fig. 6

TABLE II. Nanowire resistance evolution with annealing events. Each annealing lasts 1-3 min with an increasing operating temperature from $600^{\circ} \mathrm{C}$ for the first annealing to $800^{\circ} \mathrm{C}$ for the last.

\begin{tabular}{lcl}
\hline \hline Number of annealing & Resistance $(\mathrm{G} \Omega)$ & \multicolumn{1}{c}{ Measurement } \\
\hline 0 & 21 & resonant frequency shift under field emission \\
3 & 6.5 & resonant frequency shift under field emission \\
12 & 1.9 & resonance width vs estimation with static deflection \\
13 & 1.39 & resonance width vs estimation with static deflection \\
14 & $<1$ & resonant frequency shift under field emission \\
14 & 0.5 & IV apex/electrode contact \\
14 & 1 & resonance width vs estimation with static deflection \\
& & (serial resistance of the two nanowires) \\
\hline \hline
\end{tabular}




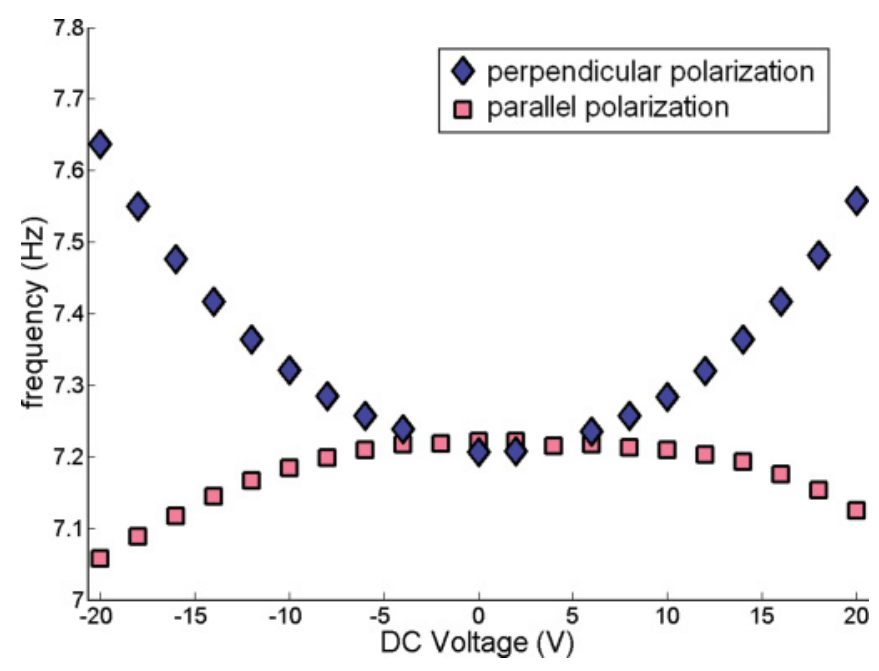

FIG. 7. (Color online) Frequency tuning with applied DC voltage for the two polarizations of the first resonant mode ( $x_{\|}$diamonds, $x_{\perp}$ squares). The difference in the curvatures comes from the electrostatic pull-in on $x_{\|}$[Eq. (13)].

in agreement with Eq. (6). There is also now an excellent agreement between the damping rate from resonance spectra and electrostatic damping from static deflection [Eq. (10)] for $R=1.39 \mathrm{G} \Omega$. The difference with the resistance found before is attributed to a resistance change after the annealing between the two experiments (see Table II).

The second series of experiments shows the influence of a resistive counter electrode being the shorter $\mathrm{SiC}$ nanowire shown in Fig. 1(b). Before the measurements, an annealing was carried out, and $R$ was found to be around $0.5 \mathrm{G} \Omega$. The nanowires had a large relative tilt angle and were to first order lying in the image plane. This arrangement allowed for observation of the two polarizations, sufficiently separated in frequency, one being approximately transverse to the image plane, $x_{\perp}$, and the other approximately parallel to the image plane, $x_{\|}$. This was useful as the electrostatic pull-in only

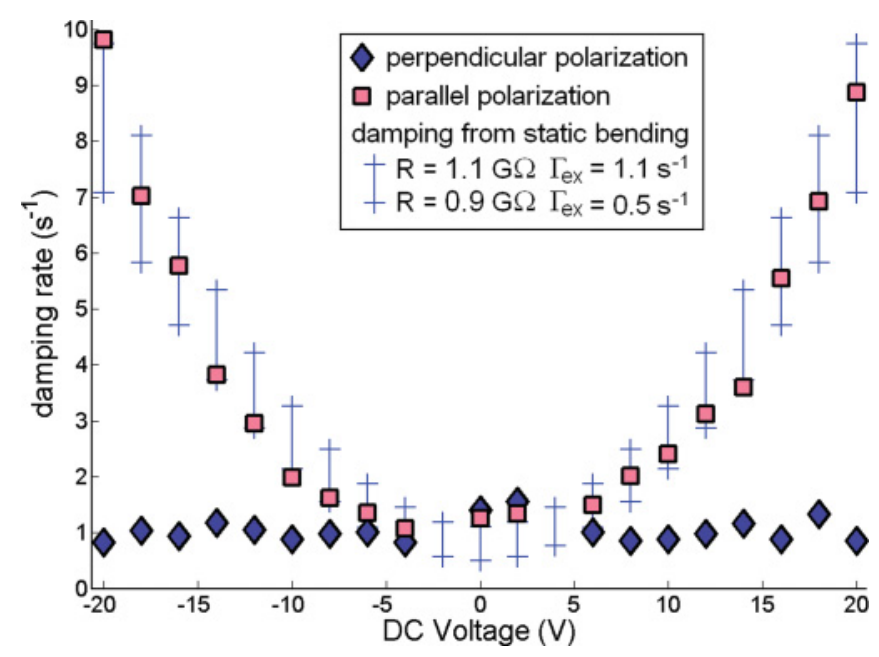

FIG. 8. (Color online) Damping rate from resonance spectra for the parallel (diamonds) and perpendicular polarization (squares). Damping rate from the conversion of the bending using Eq. (11) and a total resistance $R_{\mathrm{NW}}+R_{e}=1 \mathrm{G} \Omega$. matters for $x_{\|}$, because, to first order, $x_{\perp}$ does not change the nanowire/nanowire distance. A manifestation of this is the different frequency tuning for the two polarizations with applied voltage (see Fig. 7):

$$
\begin{aligned}
& f_{\perp}=\frac{1}{2 \pi} \sqrt{\omega_{0 \perp}^{2}-\frac{V_{d c}^{2}}{2 m_{e}} \frac{\partial^{2} C}{\partial x_{\perp}^{2}}} \\
& f_{\|}=\frac{1}{2 \pi} \sqrt{\omega_{0 \|^{2}}{ }^{2}-\frac{V_{d c}{ }^{2}}{2 m_{e}} \frac{\partial^{2} C}{\partial x_{\|}^{2}}},
\end{aligned}
$$

where the electrostatic pull-in makes $\partial^{2} C / \partial x_{\|}^{2}$ positive while $\partial^{2} C / \partial x_{\perp}^{2}$ is negative as usual for mechanical tension.

Experimental measurement of damping rate with the applied voltage shows that the electrostatic damping only affects the parallel polarization ${ }^{17}$ (see Fig. 8). An estimation of the electrostatic damping rate [Eq. (11)] is also plotted with $\partial C / \partial x_{\|}$from static deflection measurements. There is very good agreement with resonant damping rate measurements for $R=1 \mathrm{G} \Omega$. An important result here is the difference with the resistance found with the gold electrode. It comes from the significant resistance $R_{e}$ of the counter electrode that simply modifies Eq. (2) as

$$
-\frac{V_{\mathrm{NW}}}{R+R_{e}}=V_{\mathrm{DC}} C^{\prime} \frac{d x}{d t}+C \frac{d V_{\mathrm{NW}}}{d t} .
$$

For the low $T$ regime, the resistance is simply replaced by the serial resistance $R+R_{e}$.

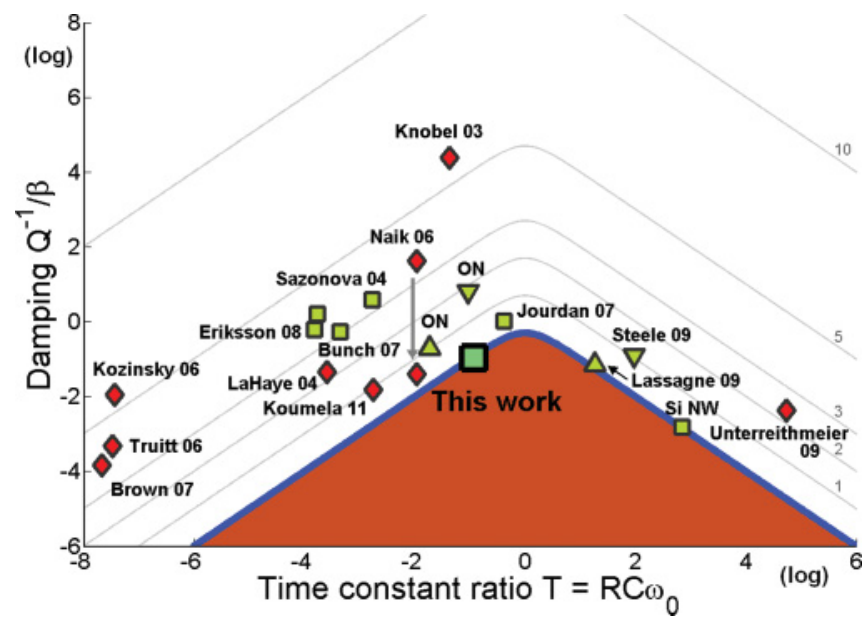

FIG. 9. (Color online) Normalized dissipation $1 / Q \beta$ as a function of the time constant ratio $T=\omega_{0} R C$ for various reported experiments (logarithmic axes). The continuous line is the universal electrostatic damping $\Gamma_{\mathrm{es}} /\left(\beta \omega_{0}\right)$ from Eq. (4). It sets a theoretical lower limitation (lower region) for the dissipation. The linear increasing and decreasing slopes correspond, respectively, to the asymptotic regime calculated in Eqs. (6) and (8). The experimental data are plotted using quality factor, capacitance ratio $\beta$, and time ratio $T$ for bottom-up (squares) (Table III) and top-down devices (diamonds) (Table IV). Lassagne $09(\triangle)$ and Steele $09(\nabla)$ data correspond to the Coulomb blockade regime. The $\mathrm{ON}$ associated triangles correspond to the Coulomb peaks when the resonator is in its conductive regime. The vertical distances from the electrostatic damping lower limit give the additional source of dissipation. 
TABLE III. Electromechanical parameters for the computation of the normalized damping $Q^{-1} / \beta$ and time ratio $T$ in Fig. 9 for bottom-up devices (normal font—parameters given by the authors or accepted(*), italics—estimated from article details). The expressions for $\beta$ and $T$ are given in Sec. III.

\begin{tabular}{|c|c|c|c|c|c|c|c|c|}
\hline Group & Frequency (MHz) & $Q$ & $m_{e}(\mathrm{~kg})$ & $R(\Omega)$ & $C(\mathrm{fF})$ & $C^{\prime}\left(\mathrm{pF} \cdot \mathrm{m}^{-1}\right)$ & bias $(\mathrm{V})$ & Ref. \\
\hline This work & 0.007 & 1500 & $5.10^{-15}$ & $1.4 \mathrm{G}$ & 1 & 1 & 10 & \\
\hline Si NW & 1 & 500 & $3.10^{-17}$ & $1 \mathrm{~T}$ & 0.1 & 1 & 400 & \\
\hline Sazonova 04 & 55 & 80 & $1.10^{-21}$ & $50 \mathrm{k}$ & 0.01 & 4 & 2.2 & 24 \\
\hline Jourdan 07 & 0.005 & 580 & $3.10^{-10}$ & $30 \mathrm{M}$ & 440 & 28000 & 0.5 & 18 \\
\hline Bunch 07 & 36 & 60 & $1.10^{-17}$ & $10 \mathrm{k}$ & 0.2 & 1000 & 2 & 25 \\
\hline \multirow[t]{2}{*}{ Eriksson 08} & 62 & 55 & $3.10^{-18}$ & $100 \mathrm{k}$ & 0.005 & 20 & 0.1 & 26 \\
\hline & 54 & 22 & $3.10^{-18}$ & $100 \mathrm{k}$ & 0.005 & 20 & 18 & \\
\hline Lassagne 09 (blocked) & 50 & 400 & $2.10^{-21}$ & $1 \mathrm{G}$ & 0.06 & $4^{*}$ & 6 & 27 \\
\hline Lassagne 09 (ON) & 50 & 100 & $2.10^{-21}$ & $1 \mathrm{M}$ & 0.06 & $4^{*}$ & 6 & \\
\hline Steele 09 (blocked) & 250 & 150000 & $2.10^{-21}$ & $1 \mathrm{G}$ & 0.06 & $4^{*}$ & 1 & 28 \\
\hline Steele $09(\mathrm{ON})$ & 250 & 2900 & $2.10^{-21}$ & $1 \mathrm{M}$ & 0.06 & $4 *$ & 1 & \\
\hline
\end{tabular}

\section{DISCUSSION}

The experimental measurements above have shown the validity of Eq. (6), which is the low $T$ regime of Eq. (5). It is important to show its validity over the full range of $T$, which is possible by considering measurements of resonators described in the literature. A large selection of resonators are described in Tables III ${ }^{18,24-28}$ and IV. ${ }^{29-35}$

It is useful to rewrite Eq. (5) as

$$
\frac{\Gamma_{t}}{\beta \omega_{0}}=\frac{\Gamma_{\mathrm{ex}}}{\beta \omega_{0}}+\frac{T}{1+T^{2}} .
$$

The experimental values of the normalized damping $\Gamma_{t} / \beta \omega_{0}=$ $Q^{-1} / \beta$ are plotted as a function of the experimental time ratio $T$ in Fig. 9. The universal ratio $T /\left(1+T^{2}\right)$ gives the lowest possible value for the total dissipation. Notice that no measurements are below this limit while six approach the curve, which is strong proof of the validity of our formula.

Other remarks can be made with respect to Fig. 9. First, the vertical distance to the universal curve gives directly the additional dissipation due to other mechanisms $\Gamma_{\mathrm{ex}} / \beta \omega_{0}$. Second, the electrostatic damping is a maximum when the mechanical and electrical time constants match $(T=1)$. In other words, the highest dissipation occurs when the resistive current $\left(V_{\mathrm{NW}} / R\right)$ and capacitive current $\left(C \omega_{0} V_{\mathrm{NW}}\right)$ have the same weight in Eq. (3). Finally, a useful engineering benchmark for NEMS devices is a simple formula for the minimum $Q$ factor due to electrostatic damping:

$$
Q_{\min }=\frac{2}{\beta} \text {. }
$$

Thus $\beta$ appears to be a "damping parameter," which would seem to be in contradiction with it being termed a coupling strength. In fact, it can play either role depending on $T$, i.e., for $T \gg 1$ it functions as a coupling strength in that it shifts the resonant frequency [Eq. (9)].

Several aspects in the figure should be examined in some detail. The results in Ref. 18 (Jourdan 07) are noteworthy because the resonator functioned at low $T$, the result is close to the universal curve, and it was the only reference that showed that electrostatic damping was the main mechanism. In Ref. 31 (Naik 06), the resonator has a single electron transistor as counter-electrode, and the damping was varied by increasing $V_{\mathrm{DC}}$. Our plot shows that a significant part of the dissipation can be explained by this classical description of the electromechanical coupling. In Ref. 26 (Eriksson 08), the quality factor was shown to decrease substantially with $V_{\mathrm{DC}}$. However, Fig. 9 shows that the associated $Q^{-1} / \beta$ varied little, which means that electrostatic damping is not the principal loss mechanism. It may be related to near-field experiments with cantilevers oscillating parallel to surfaces where the electrostatic origin of the dissipation has been demonstrated, but the exact nature and mathematical formulation is not clear. $^{36,37}$

TABLE IV. Electromechanical parameters for top-down devices (normal font-parameters given by the authors, italics—estimated from

\begin{tabular}{|c|c|c|c|c|c|c|c|c|}
\hline Group & Frequency (MHz) & $Q$ & $m_{e}(\mathrm{~kg})$ & $R(\Omega)$ & $C(\mathrm{fF})$ & $C^{\prime}\left(\mathrm{pF} \cdot \mathrm{m}^{-1}\right)$ & Bias (V) & Ref. \\
\hline Knobel 03 & 117 & 1700 & $3.10^{-15}$ & $100 \mathrm{k}^{*}$ & 0.6 & 30 & 5 & 29 \\
\hline Lahaye 04 & 19.7 & 50000 & $1.10^{-15}$ & $70 \mathrm{k}^{*}$ & 0.03 & 30 & 15 & 30 \\
\hline \multirow[t]{2}{*}{ Naik 06} & 21.9 & 120000 & $6.10^{-16}$ & $200 \mathrm{k}^{*}$ & 0.4 & 300 & 0.1 & 31 \\
\hline & 21.9 & 13000 & $6.10^{-16}$ & $200 \mathrm{k}^{*}$ & 0.4 & 300 & 10 & \\
\hline Truitt 06 & 11 & 30000 & $1.10^{-15}$ & 10 & 0.05 & 300 & 15 & 32 \\
\hline Kozinsky 06 & 8.6 & 2500 & $3.10^{-16}$ & 10 & 0.07 & 80 & 20 & 17 \\
\hline Brown 07 & 0.007 & 20000 & $2.10^{-9}$ & 5 & 100 & 8000 & 50 & 33 \\
\hline Unterreithmeier 09 & 8.9 & 150000 & $2.10^{-15}$ & $>\mathrm{T}$ & 1 & 3000 & 1 & 34 \\
\hline Koumela 11 & 1195 & 2000 & $7.10^{-17}$ & $100 \mathrm{k}$ & 0.03 & 250 & 20 & 35 \\
\hline
\end{tabular}
article details). Entries with $*$ include the counter electrode resistance. 
In general there are a few measurements that correspond to $T>1$. References 27 and 28 (Lassagne 09 and Steele 09) concern electromechanical coupling of carbon nanotube resonators exhibiting Coulomb blockade. The high $T$ regime is reached because of the large resistances expected in the Coulomb blockade regime $[\sim \mathrm{G} \Omega$, up (Lassagne 09) and down (Steele 09) triangle]. As the conductance oscillates with the gate voltage, the equations for electrostatic damping when $T \gg 1$ describe well the observed damping rate [Eq. (8)] and frequency shift [Eq. (9)]. Very high quality factors (150 000) are reported in Ref. 34 (Unterreithmeier 10) with insulating resonators $(R>\mathrm{T} \Omega)$. The corresponding data in Fig. 9 considers a lower value of $T$, i.e., when $R=1 \mathrm{~T} \Omega$ ). This confirms that no dissipative current is induced in the high $T$ regime. Our recent measurements of $\Gamma_{t} / \beta$ for high purity $\mathrm{Si}$ nanowires $(\mathrm{Si} \mathrm{NW})$ with resistances $\sim \mathrm{T} \Omega$ fall precisely on the universal curve.

\section{CONCLUSION}

We have experimentally shown that the damping of a cantilever can be dominated by ohmic losses induced by the electromechanical coupling between charge and mechanical displacement. This was done by varying the geometry of the capacitive environment and the applied voltage in several dedicated experiments and comparing it to a theoretical model. The experiments include an original approach for determining the resonant damping rate using only static measurements that in turn gives a good estimate of the cantilever resistance. The developed model is characterized by a simple universal formula involving two dimensionless parameters widely applicable to NEMS devices. It is in agreement with data collected in a wide literature survey and quantifies the contribution of electrostatic damping to the total damping.
${ }^{1}$ K. L. Ekinci and M. L. Roukes, Rev. Sci. Instrum. 76, 061101 (2005).

${ }^{2}$ P. S. Waggoner and H. G. Craighead, Lab Chip 7, 1238 (2007).

${ }^{3}$ Mo Li, H. X. Tang, and M. L. Roukes, Nature Nanotechnology 2, 114 (2007).

${ }^{4}$ K. L. Ekinci, X. M. H. Huang, and M. L. Roukes, Appl. Phys. Lett. 84, 4469 (2004).

${ }^{5}$ K. Jensen, Kwanpyo Kim, and A. Zettl, Nature Nanotechnology 3, 533 (2008).

${ }^{6}$ P. Mohanty, D. A. Harrington, K. L. Ekinci, Y. T. Yang, M. J. Murphy, and M. L. Roukes, Phys. Rev. B 66, 085416 (2002).

${ }^{7}$ R. Lifshitz and M. L. Roukes, Phys. Rev. B 61, 5600 (2000).

${ }^{8}$ R. Lifshitz, Phys. B: Cond. Matt. 316-317, 397 (2002).

${ }^{9}$ R. B. Bhiladvala and Z. J. Wang, Phys. Rev. E 69, 036307 (2004).

${ }^{10}$ J. A. Judge, D. M. Photiadis, J. F. Vignola, B. H. Houston, and J. Jarzynski, J. Appl. Phys. 101, 013521 (2007).

${ }^{11}$ A. N. Cleland and M. L. Roukes, Sens. Actuators A 72, 256 (1999).

${ }^{12}$ K. R. Brown, J. Britton, R. J. Epstein, J. Chiaverini, D. Leibfried, and D. J. Wineland, Phys. Rev. Lett. 99, 137205 (2007).

${ }^{13}$ S. O. R. Moheimani, IEEE Cont. Sys. Tech. 11, 482 (2003).

${ }^{14}$ W. J. Choi, Y. Jeon, J.-H. Jeong, R. Sood, and S. G. Kim, J. Electroceram 17, 543 (2006).

${ }^{15} \mathrm{~V}$. A. Sazonova, A tunable carbon nanotube resonator, Ph.D. thesis, Cornell University (2006).

${ }^{16} \mathrm{~S}$. T. Purcell, P. Vincent, C. Journet, and Vu Thien Binh, Phys. Rev. Lett. 89, 276103 (2002).

${ }^{17}$ I. Kozinsky, H. W. Ch. Postma, I. Bargatin, and M. L. Roukes, Appl. Phys. Lett. 88, 253101 (2006).

${ }^{18}$ G. Jourdan, G. Torricelli, J. Chevrier, and F. Comin, Nanotechnology 18, 475502 (2007).

${ }^{19}$ M. Choueib, A. Ayari, P. Vincent, M. Bechelany, D. Cornu, and S. T. Purcell, Phys. Rev. B 79, 075421 (2009).

${ }^{20}$ A. Ayari, P. Vincent, S. Perisanu, M. Choueib, V. Gouttenoire, M. Bechelany, D. Cornu, and S. T. Purcell, Nano Lett. 7, 2252 (2007).
${ }^{21}$ D. W. Dye, Proc. Phys. Soc. London 38, 399 (1925).

${ }^{22}$ E. Buks and M. L. Roukes, Phys. Rev. B 63, 033402 (2001).

${ }^{23}$ P. Vincent, S. Perisanu, A. Ayari, M. Choueib, V. Gouttenoire, M. Bechelany, A. Brioude, D. Cornu, and S. T. Purcell, Phys. Rev. B 76, 085435 (2007).

${ }^{24}$ V. Sazonova, Y. Yaish, H. Üstünel, D. Roundy, T. A. Arias, and P. L. McEuen, Nature (London) 431, 284 (2004).

${ }^{25}$ J. S. Bunch, A. M. van der Zande, S. S. Verbridge, I. W. Frank, D. M. Tanenbaum, J. M. Parpia, H. G. Craighead, and P. L. McEuen, Science 315, 490 (2007).

${ }^{26}$ A. Eriksson, S. Lee, A. A. Sourab, A. Isacsson, R. Kaunisto, J. M. Kinaret, and E. E. B. Campbell, Nano Lett. 8, 1224 (2008).

${ }^{27}$ B. Lassagne, Y. Tarakanov, J. Kinaret, D. Garcia-Sanchez, and A. Bachtold, Science 325, 1107 (2009).

${ }^{28}$ G. A. Steele, A. K. Hüttel, B. Witkamp, M. Poot, H. B. Meerwaldt, L. P. Kouwenhoven, and H. S. J. van der Zant, Science 325, 1103 (2009).

${ }^{29}$ R. G. Knobel and A. N. Cleland, Nature (London) 424, 291 (2003).

${ }^{30}$ M. D. LaHaye, O. Buu, B. Camarota, and K. C. Schwab, Science 304, 74 (2004).

${ }^{31}$ A. Naik, O. Buu, M. D. LaHaye, A. D. Armour, A. A. Clerk, M. P. Blencowe, and K. C. Schwab, Nature (London) 443, 193 (2006).

${ }^{32}$ P. A. Truitt, J. B. Hertzberg, C. C. Huang, K. L. Ekinci, and K. C. Schwab, Nano Lett. 7, 120 (2006).

${ }^{33}$ K. R. Brown, J. Britton, R. J. Epstein, J. Chiaverini, D. Leibfried, and D. J. Wineland, Phys. Rev. Lett. 99, 137205 (2007).

${ }^{34}$ Q. P. Unterreithmeier, E. M. Weig, and J. P. Kotthaus, Nature (London) 458, 1001 (2009).

${ }^{35}$ A. Koumela, D. Mercier, V. Gouttenoire, C. Marcoux, S. T. Purcell, and L. Duraffourg, Proc. Eurosensors XXV (2011).

${ }^{36}$ B. C. Stipe, H. J. Mamin, T. D. Stowe, T. W. Kenny, and D. Rugar, Phys. Rev. Lett. 87, 096801 (2001).

${ }^{37}$ B. Gotsmann, Nat. Mater. 10, 87 (2011). 